\title{
Investigating a DTV-based physical activity application to facilitate wellbeing in older adults
}

\author{
Alex Carmichael \\ School of Computing \\ University of Dundee, Dundee, DD1 4HN \\ \{acarmichael; mrice\}@computing.dundee.ac.uk
}

\author{
Freya MacMillan \\ Dept. of Sport, Culture \& the Arts \\ University of Strathclyde, Glasgow, G13 1PP \\ \{freya.macmillan; alison.kirk\}@strath.ac.uk
}

\begin{abstract}
This paper describes a study that examined the usability and acceptability of a physical exercise application using the digital television platform for older adults. A key aim of this study was to obtain feedback from representative older users to inform further development of this application. Other key outcomes were to measure physical exertion objectively and subjectively whilst using this application and to investigate potential acute effects of the session on cognitive function and mood. The results indicated that the settings used for the current exercises did not produce physical exertion at a high enough intensity to have significant health benefits, although a positive impact on mood was found. Subjective feedback identified a number of issues regarding various application features and possible adaptations for the future design and development of this application, including the use of gesture based user-control and the potential importance of monitoring user heart-rate to set exercise parameters.
\end{abstract}

Older adults, physical exercise, DTV, gesture control, Wii mote.'

\section{INTRODUCTION}

Physical activity is known to provide older adults with substantial benefits to their physical and mental wellbeing, including improvements in cardiovascular function, balance, muscle strength and endurance, as well as general affect (mood) and cognitive status. Current guidelines recommend that older adults should undertake a minimum of 150 minutes of moderate physical exercise a week [11]. However, there is evidence that only about one third of the older population currently meet these guidelines [14]. This is a significant issue, given that in the UK alone, cardiovascular disease is responsible for the deaths of over 200,000 people per year, with $3 \%$ of all deaths directly attributed to inadequate physical activity [2]. Important barriers to physical exercise for many older adults include: limited motivation, fear of injury, or other medical conditions, particularly those affecting mobility. However, while various initiatives currently promote structured exercise programmes at gyms or community centres, low levels of attendance and high drop-out rates remain a formidable problem, with about half of all (initially sedentary) adults quitting within the first six months [12]. Thus, it is likely that many older adults could benefit from a suitably designed physical activity programme tailored to their requirements and easily accessible within their home.

The present switchover of analogue to digital television (DTV) across Europe means that households nationwide will require digital reception equipment within the next few years. As such, the switchover presents a great opportunity to harness the technical capabilities of next generation digital receivers and dynamic broadcasting platforms to support the delivery of an easy-to-use physical activity programme without the need for a PC or games console. This includes the incorporation of advanced pointing and gesture-type devices that are currently emerging in the DTV market (e.g. the Hillcrest Loop ${ }^{1}$ and the Gryation in-air remote $^{2}$ ). However, research into the use of computer-based tools to promote effective physical activity for older adults is limited.

Despite this, over the past two decades there have been a number of studies that have explored the use of video games for older adults to support cognitive functions [e.g. 8, 17]. Recently, this has included a research study by Basak et al. [3], which found significant cognitive benefits in the training of a PC strategy game, particularly amongst executive control functions in older adults. More broadly however, when it comes to exploring physical exertion, studies have typically involved children, young fit adults or specialised population groups (e.g. persons with traumatic brain injury), and most have reported only on energy expenditure during play. Consequently, to our knowledge, very few studies have been published using older adults. One notable example is a study by Schuler et al. [13] which has briefly reported on the positive effects of using the Nintendo Wii bowling with older adults over

\footnotetext{
${ }^{1}$ http://www.hillcrestlabs.com/

2 http://www.gyration.com/index.php/us/products/in-airremotes.html
} 
a single gaming session.

Looking more widely at 'exer-gaming' research, Graves et al. [9] compared predicted energy expenditure during a sedentary computer game with three active games for the Nintendo $\mathrm{Wii}^{\mathrm{TM}}$. Involving 11 physically active children aged $13-15$ years; each game was played for 15 minutes with a 5 minute rest period between games. The results showed that the active games resulted in an increase in energy expenditure of $60 \mathrm{kcal}$ per hour compared to the sedentary one. While, an undergraduate study of 12 highly active adults, mean age $22.1 \pm 2.0$ years, found that the intensitylevel of the Wii boxing and tennis games met the American College of Sports Medicine (ACSM) minimal recommendation for weekly expenditure [4]. Both studies used the Nintendo Wii Sports as distinct from the Nintendo Wii Fit (NWF).

Driven by such studies and the commercial success of related gaming technologies, the Department of Health and NHS in the UK have recently promoted the NWF to combat sedentary lifestyles amongst obese population groups, particularly children [5]. However, we have identified that many aspects of the general design, range, and approach to the physical activities the NWF provides, makes the device unsuitable for many older adults [10]. To address this, we explored the design of a more ageappropriate physical activity programme for older adults as part of the EC funded Vital Mind project. The aim of the present pilot study was to gather feedback from representative older users regarding issues of usability, acceptability, and the potential long-term use of this alternative approach. Other objectives were to measure physical exertion during the exercise program, both objectively and subjectively, in order to determine if this application could be used as a physical activity tool to help older adults reach the current physical activity recommendations for health benefits. Cognitive function and mood were also assessed to investigate the possible acute effects of using the application on these outcomes. This paper will describe in detail the software programme developed for the study and the hardware set-up. The procedure and experimental method are then discussed, followed by detailed findings from the quantitative and qualitative elements of the study. We finish by summarising our study and highlighting some key issues for future research.

\section{PHYSICAL ACTIVITY PROGRAMME}

Twenty-four exercises were developed to run as part of a thirty minute TV-based training programme. These exercises were carefully designed to include a full range of upper and lower body movements, and were based on recognised exercise programs designed for older adults, which the research team were familiar with. Strong emphasis was placed on giving the user clear instructions in order to ensure they could carry out the exercises appropriately. This included a focus on giving the user some encouragement, without introducing the element of competiveness common amongst NWF games.

Beginning with warm-up activities, the user then moved onto sections involving aerobic and muscular exercises. The exercises in this section were interleaved with a 'march-on-the-spot' activity to avoid cooling down during the instruction period. Finally, the programme finished with a cool-down and flexibility component. Also included was a five minute animated introduction at the start of the programme explaining the purpose and benefits of the 'virtual instructor' and visually demonstrating interaction using the Wii mote.

With the long term aim of exploring the development of a specialised gesture control, this pilot work used the integrated features of the Wii mote to support the detection of a broad range of physical movements. This was the only input device used for the study. However, once software development was underway, it was discovered that recognition of the real-world gestures was somewhat error prone. Thus some of exercises necessitated the user to press a key to count repetitions.

Presented in table 1 is a summary of all the sequenced activities in the programme, including the duration of each activity timed in seconds. During the performance, each exercise was either controlled by a button press (A or B) or physical gesture (no buttons). As illustrated in the table, for activities like the wrist circles, the exercise was repeated twice in the program (e.g. $2 \times 30$ s) to individually exercise each wrist. Prompts in the programme automatically instructed users when to change the Wii mote into a different hand.

For the user interface (UI), each exercise consisted of two stages: a) a short exercise demonstration, and, b) a physical workout of that particular exercise. The instructions consisted of highlighted text with accompanying audio commentary, as well as a short one minute (looped) video demonstration of a virtual instructor performing the exercise to be undertaken (see figure 1). In the design, careful attention was paid to refine the instructions in order to keep them short, informative, and to the point. Similarly, the design of the UI took into consideration interacting in the television environment by ensuring; good screen contrast, appropriate text legibility and layout of screen content for the target group. 


\begin{tabular}{|c|c|c|c|}
\hline & Activity description & Input modality & $\begin{array}{c}\text { Duration } \\
\text { of } \\
\text { activity }\end{array}$ \\
\hline \multicolumn{4}{|c|}{ Warm up section } \\
\hline 1 & Heel digs & Button press (A) & $30 \mathrm{~s}$ \\
\hline 2 & Side steps & Button press (A) & $30 s$ \\
\hline 3 & Hamstring curls & Button press (A) & $30 s$ \\
\hline 4 & Wrist circles & Button press (A) & $2 \times 30 s$ \\
\hline 5 & Ankle circles & Button press (B) & $2 \times 30 s$ \\
\hline 6 & Shoulder circles & Button press (B) & $30 \mathrm{~s}$ \\
\hline \multicolumn{4}{|c|}{ Aerobic section } \\
\hline 1 & $\begin{array}{c}\text { Heel dig / bicep } \\
\text { curl }\end{array}$ & Gesture & $30 s$ \\
\hline 2 & $\begin{array}{c}\text { Side step / upright } \\
\text { rowing }\end{array}$ & Gesture & $30 s$ \\
\hline 3 & $\begin{array}{l}\text { Marching on the } \\
\text { spot }\end{array}$ & Gesture & $30 s$ \\
\hline 4 & $\begin{array}{l}\text { Knee lift / chest } \\
\text { press }\end{array}$ & Gesture & $30 \mathrm{~s}$ \\
\hline 5 & $\begin{array}{l}\text { Hamstring curl / } \\
\text { rowing }\end{array}$ & Gesture & $30 s$ \\
\hline 6 & Half jacks & Gesture & $30 s$ \\
\hline \multicolumn{4}{|c|}{ Muscular section } \\
\hline 1 & Bicep curl & Gesture & $30 s$ \\
\hline 2 & Triceps extension & Gesture & $30 s$ \\
\hline 3 & Sit to stand squat & Button press (A) & $30 s$ \\
\hline 4 & Side bend & Button press (A) & $2 \times 30 s$ \\
\hline \multicolumn{4}{|c|}{ Cool down section } \\
\hline 1 & Heel dig & Button press (A) & $30 s$ \\
\hline 2 & Side step & Button press (A) & $30 s$ \\
\hline 3 & Hamstring curl & Button press (A) & $30 s$ \\
\hline \multicolumn{4}{|c|}{ Stretches } \\
\hline 1 & Hamstring stretch & Button press (A) & $2 \times 30 s$ \\
\hline 2 & Calf stretch & Button press (A) & $2 \times 30 s$ \\
\hline 3 & Chest stretch & Button press (A) & $30 s$ \\
\hline 4 & Quadriceps stretch & Button press (A) & $2 \times 30 s$ \\
\hline 5 & $\begin{array}{c}\text { Shoulder / triceps } \\
\text { stretch }\end{array}$ & Button press (A) & $30 \mathrm{~s}$ \\
\hline
\end{tabular}

Table 1: Description of the exercise program.

During these instructions, users had the opportunity to practice the exercise, for example, to ensure they were holding the input device correctly. When the user felt ready, they pressed the ' 1 ' key and the exercise stage would begin.

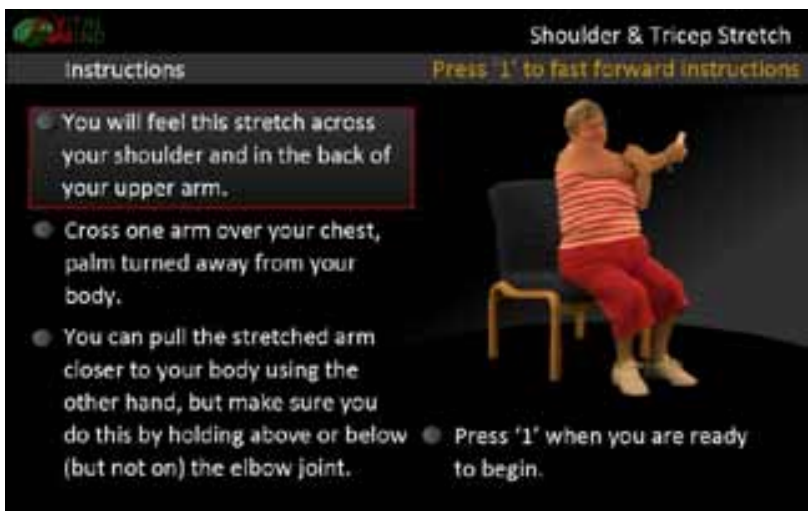

Figure 1: Screen shot of the instructions. Left, bullet pointed information. Right, video of the virtual instructor.

To engage in the physical workout, the UI consisted of a number of key features that were designed to make the activities fun, engaging and simple to use. As illustrated in figure 2, these included:

1. On-screen confirmation: to begin exercising, a confirmation box with a countdown automatically appeared in the centre of the screen, while at the end of the exercise, additional instructions told the user when to stop. Some of the exercises involved movement alternating between different parts of the body; again relevant prompts were provided as required.

2. Virtual Instructor: Chosen to be age-appropriate, the 'coach' was positioned within the centre of the screen, and was designed to visually reinforce the movements required for that particular exercise. Depending on the particular movements involved (including sitting or standing) the views of the 'coach' presented to the user comprised of a mixture of different camera angles to ensure clarity.

3. Background music: to accompany the virtual instructor, and help users keep in beat with the movement of the exercise, a selection of aerobic music was played at a tempo that is comparable with that of the exercise in question. Tempo during the warm-up, aerobics and cool-down sections ranged from 130-134bpm whilst during the muscular section it was between 118-120bpm. Slower and less overtly rhythmic music was used for the stretching component.

4. Live video (of the user): with the audio disabled, this was implemented to allow the user to selfcorrect their body position, particularly in comparing their performance with the instructor's (an analogue to a large mirror often used at exercise classes).

5. Repetition count: as an indication of how individuals were progressing, when the full movement was detected by the system (or key pressed by the user), an eye-catching star rotated 
with each count, within which the repetition-count number increased incrementally.

6. Additional instructions: text prompts were displayed at the bottom of the screen (and duplicated in audio) to restate posture and position (eg 'reach up and over with your arm' or 'keep your feet flat on the floor'). These prompts were based upon the research team's experience of running similar exercises with older adults and the common errors novice exercisers make. In addition, throughout the exercises, words of encouragement were intermittently presented by the programme, such as 'looking good' and 'well done'.

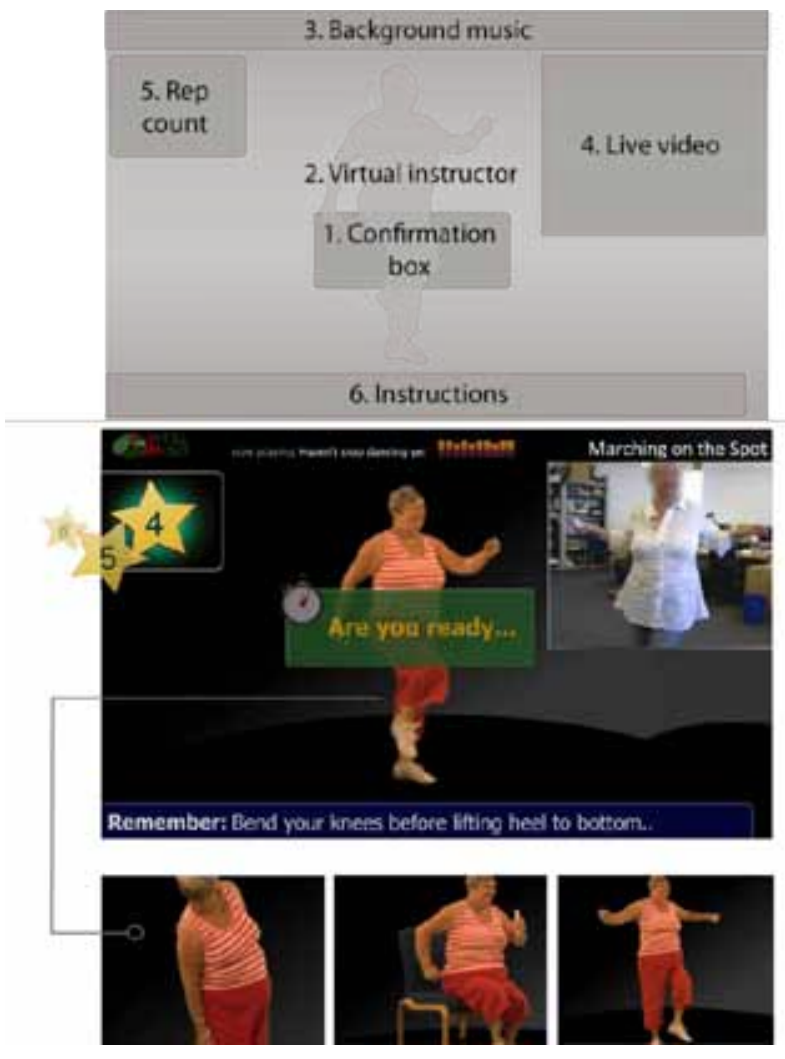

Figure 2: Screen shot of the exercising interface.

As part of the preparation for the software development process, for the inclusion of the virtual instructor, a green screen studio was utilised to record the full set of exercises. An older volunteer aged 73 from a local exercise class volunteered to be the coach. Within the studio, two HD cameras were set-up to simultaneously record different positions (front and side) of each exercise, and careful attention was paid to the use of even lighting in the studio to avoid excessive shadows. Once complete, the videos were then edited to key out the chroma background, and segment the footage into single exercises (combining the video from different camera angles). Furthermore, as all text in the programme was duplicated by audio commentary, all the audio was recorded in a professional sound proof studio, using the voice of an aerobics instructor with a local accent and up-beat tone.

For user testing, interactive prototypes were displayed on a 26" wide-screen LCD television connected to a mini-PC unit $(1.6 \mathrm{GHz})$ with a built in graphics card. Compared to the architecture of many set-top boxes, a relatively powerful CPU processor was required to smoothly run the prototype multimedia content, which was not compressed in the same way digital transmissions are. Also, given variations in colour configuration, text display and a lack of support for video capturing devices like the USB web- camera, a PC simulator appeared an appropriate option as a platform for the prototype system used in this study

Supported on many consumer electronic devices, the UI was primarily built in Flash Lite $®$ (version 3.1), developed using Adobe $®$ Flash $®$ Professional CS4. As part of the software architecture, ActionScript (.as), flash video (.flv), sound (.wav) and XML files were externally loaded into the flash movie from a local directory. This was purposely done to reuse much of the scripting code, and reduce memory demands at runtime, caused by embedding large files within the Flash time line. To enable the Wii mote to communicate with the Flash application, a proprietary Bluetooth stack (BlueSoleil ${ }^{\mathrm{TM}} 6.4^{3}$ ) was required to detect and pair the peripheral device to a local human interface driver (HID). As the testing PC was not Bluetooth enabled, a standard USB 2.0 Bluetooth adaptor was used to support wireless connectivity. For gesture control, an application called GlovePIE ${ }^{\text {TM }}{ }^{4}$ was used to emulate the Wii mote. Within this environment, a software-based program was specifically developed to control the $x$, $y$ and $z$ accelerations, and to handle a small set of button commands (i.e. the A, B, and 1 buttons) on the Wii mote. As part of the motion sensing, coordinated gestures were then translated into the motion sensing buttons in order to be used as navigational commands in the UI application. However, as intricate gestures and lower body movements were difficult to accurately detect, for some exercises (see table 1), users were required to press a single button after each completed repetition. We considered this compromise to be acceptable for the current study, but recognise that it would not be for a finalised system.

\section{METHOD}

Nineteen participants were recruited via the University of Strathclyde (4 male, 15 female). All participants were aged $>55$ years (Mean age 62.5 years), and provided written consent to take part in the study. Each trial session lasted between 60-90 minutes and was conducted in a laboratory setting.

\footnotetext{
${ }^{3}$ http://www.bluesoleil.com/

${ }^{4}$ http://sites.google.com/site/carlkenner/glovepie
} 


\subsection{Pre-session measures}

Blood pressure, height and weight were measured prior to the physical activity sessions and body mass index (BMI) calculated $(\mathrm{kg} / \mathrm{m} 2)$. Mood was also assessed using the Positive and Negative Affect Schedule (PANAS) [16] which consists of 24 items designed to rate (on a 5-point Likert scale) how participants felt over the past month. The PANAS has two subscales; a positive and negative scale (PANASpos and PANASneg respectively). Scores are added to give total PANASpos and PANASneg scores. The greater the PANASpos total and lower the PANASneg total the more favourable the overall mood of the individual. Thus an increase in PANASpos and decrease in PANASneg represents an overall improvement in mood, whilst a decrease in PANASpos and increase in PANASneg indicates a decline.

Cognitive function was also assessed using a test of non-verbal intelligence (TONI-3). This test comes with two parallel forms (A and $B$ ) making it suitable for pre- and post-testing, as forms $A$ and $B$ were counterbalanced over these sessions. The TONI-3 measures aptitude, abstract reasoning, and problem solving and consists of 45 items each with a matrix of images and a problem to solve [7]. Five training items are used to explain the task. A facilitator noted the participants' answer for each of the items. Scores are calculated by totalling the number of correct answers (until 3 out of 5 consecutive items are answered incorrectly). For the TONI-3, it was expected that administrating this test would identify if physical activity had an impact on participants' skills at solving novel abstract problems (a prominent component of intelligence). However, it is important to note that these tests were only designed to take a generalised view of any marked improvements in intelligence.

During the sessions, heart rate was measured using a Polar heart monitor and perceived exertion was recorded using Borg's Perceived Exertion scale (RPE) at the end of each activity [6]. Predicted heart rate maximum for each individual was determined using the equation 208-(0.7xage) [15] with the predicted heart rate being rounded down to the nearest whole number. RPE was used to determine if users perceived they were working at the same level as their actual heart rate. In order to categorise heart rate intensity, average and maximum heart rate during the session was recorded in beats per minute and expressed as a percentage of predicted heart rate maximum (\%HRmax). Immediately after each individual activity, heart rate was noted and \%HRmax calculated. Heart rate and RPE during the physical activity session were categorised by intensity using the ACSM guidelines. Briefly, working between 50-60\%HRmax (RPE 9) corresponds to a 'light' intensity, $64-76 \% \mathrm{HRmax}$ is a 'moderate' intensity
(RPE 11), 77-93\%HRmax (RPE 13) is a 'vigorous' intensity with 94\%HRmax (RPE 15) relating to a 'very hard' intensity. An RPE rating between 12 and 16 corresponds to the intensity levels required to gain physiological adaptation [1].

\subsection{Post-session measures}

Participants completed the PANAS based on how they felt immediately after the physical activity session. Cognitive function was assessed using the TONI-3 with the appropriate parallel set of items. Questions regarding the application's usability and acceptability were given using a short questionnaire, followed by a 15 minute semi-structured interview with each participant.

\subsection{Data analysis}

Changes in PANAS positive and negative subscale scores and TONI-3 score for pre and post sessions were analysed. The normality of the distributions was assessed using the Kolmogorov-Smirnov test. For normally distributed data $(p>0.05)$ paired-sample t-tests were used to find changes between pre and post-session measures. When data was not normally distributed $(p<0.05)$ a Wilcoxon signed-rank test was used to determine differences between pre and post session values. Significance was set at $p<0.05$.

From the questionnaires and interviews the following topics were covered: enjoyment and difficulty rating of the activities, usability of the Wii mote, appropriateness of the activities (including the use of instructions and background music), and future modifications in the application. Interview transcripts were read and main themes established. Participant quotes relevant to each theme were then tabulated together and divided into smaller sub- themes.

\section{RESULTS}

Over the full session, average heart rate was found to be of light intensity $(57.18 \pm 5.8 \% \mathrm{HRmax})$ and maximum heart rate was at a moderate level of intensity $(76.30 \pm 12.26 \% \mathrm{HRmax}$, although this is close to the 'vigorous' level). The mean intensity for each individual activity is shown in table 2 as \%HRmax (actual intensity) and RPE (perceived intensity). Three of the aerobic exercises corresponded to a moderate intensity; knee lift with chest press arms, hamstring curl with upright row arms and half jacks. All other activities were of a light intensity based on the heart rate data. For RPE, two exercises fell into the range required for physiological adaptation (hamstring curl with upright row arms and half jacks), with the remaining activities having a mean RPE value below this range (RPE 12-16) [1]. 
Table 2: Mean perceived (RPE) and actual (\%HRmax) intensity for each of the activities $(n=19)$.

\begin{tabular}{|c|c|c|c|}
\hline Activity & Description & $\begin{array}{c}\text { Mean } \\
\text { \%HRmax } \\
\text { (土SD) }\end{array}$ & $\begin{array}{c}\text { Mean } \\
\text { RPE ( } \pm S D)\end{array}$ \\
\hline \multicolumn{4}{|c|}{ Warm up } \\
\hline 1 & Heel digs & $56.11( \pm 5.47)$ & $8.32( \pm 1.89)$ \\
\hline 2 & Side steps & $53.41( \pm 6.02)$ & $8.74( \pm 1.82)$ \\
\hline 3 & Hamstring curls & $59.73( \pm 8.71)$ & $9.47( \pm 1.81)$ \\
\hline 4 & Wrist circles & $53.42( \pm 8.39)$ & $8.95( \pm 1.75)$ \\
\hline 5 & Ankle circles & $52.63( \pm 5.20)$ & $9.32( \pm 1.95)$ \\
\hline 6 & Shoulder circles & $54.26( \pm 7.09)$ & $9.58( \pm 2.06)$ \\
\hline \multicolumn{4}{|c|}{ Aerobic section } \\
\hline 1 & $\begin{array}{c}\text { Heel digs/bicep } \\
\text { curls }\end{array}$ & $60.00( \pm 5.82)$ & $10.26( \pm 1.70)$ \\
\hline 2 & $\begin{array}{l}\text { Side steps/ } \\
\text { upright rows }\end{array}$ & $61.4( \pm 9.17)$ & $10.47( \pm 1.71)$ \\
\hline 3 & $\begin{array}{c}\text { Marching on the } \\
\text { spot }\end{array}$ & $63.30( \pm 8.76)$ & $11.16( \pm 1.83)$ \\
\hline 4 & $\begin{array}{c}\text { Knee lifts/chest } \\
\text { press }\end{array}$ & $69.67( \pm 9.02)$ & $11.84( \pm 1.57)$ \\
\hline 5 & $\begin{array}{c}\text { Hamstring curl/ } \\
\text { rows }\end{array}$ & $69.09( \pm 9.80)$ & $12.16( \pm 1.50)$ \\
\hline 6 & Half jacks & $69.16( \pm 7.53)$ & $12.32( \pm 1.29)$ \\
\hline \multicolumn{4}{|c|}{ Muscle section } \\
\hline 1 & Bicep curls & $56.98( \pm 1.87)$ & $10.74( \pm 1.66)$ \\
\hline 2 & $\begin{array}{c}\text { Triceps } \\
\text { extensions }\end{array}$ & $57.13( \pm 6.53)$ & $10.78( \pm 1.78)$ \\
\hline 3 & $\begin{array}{l}\text { Sit to stand } \\
\text { squat }\end{array}$ & $57.42( \pm 6.06)$ & $11.11( \pm 1.63)$ \\
\hline 4 & Side bends & $53.64( \pm 6.81)$ & $10.79( \pm 1.87)$ \\
\hline \multicolumn{4}{|c|}{ Cool-down } \\
\hline 1 & Heel digs & $58.45( \pm 1.47)$ & $11.11( \pm 1.76)$ \\
\hline 2 & Side steps & $56.83( \pm 1.85)$ & $11.21( \pm 1.40)$ \\
\hline 3 & Hamstring curls & $62.29( \pm 8.75)$ & $11.47( \pm 1.31)$ \\
\hline \multicolumn{4}{|c|}{ Stretches } \\
\hline 1 & $\begin{array}{l}\text { Hamstring } \\
\text { stretch }\end{array}$ & $58.90( \pm 6.01)$ & $11.06( \pm 2.04)$ \\
\hline 2 & Calf stretch & $57.87( \pm 5.88)$ & $10.50( \pm 2.36)$ \\
\hline 3 & Chest stretch & $59.12( \pm 7.99)$ & $9.94( \pm 2.04)$ \\
\hline 4 & $\begin{array}{l}\text { Quadriceps } \\
\text { stretch }\end{array}$ & $59.40( \pm 6.39)$ & $10.61( \pm 2.38)$ \\
\hline 5 & $\begin{array}{c}\text { Shoulder/triceps } \\
\text { stretch }\end{array}$ & $59.23( \pm 6.58)$ & $10.39( \pm 2.30)$ \\
\hline
\end{tabular}

Change in mean mood and cognitive function scores pre to post session are shown in table 3. Mood was found to improve after the session with an increase in PANAS positive score $(z=-2.63, r=-0.60, p$ $=0.008)$ and an equivalent decrease in PANAS negative score $(z=-2.68, r=-0.62, p=0.007)$. No significant difference in TONI-3 score was found before and after the session $(z=-0.94, r$ $=-0.22, p=0.347$ ).

Table 3: Change in average PANAS and TONI-3 measures pre to post session $(n=19)$.

\begin{tabular}{|c|c|c|c|}
\hline $\begin{array}{c}\text { Outcome } \\
\text { measu re }\end{array}$ & $\begin{array}{c}\text { P re-session } \\
\text { mean } \pm \text { SD }\end{array}$ & $\begin{array}{c}\text { Post-session } \\
\text { mean } \pm \text { SD }\end{array}$ & Sig. \\
\hline PANASpos & $45.53 \pm 10.52$ & $49.11 \pm 12.37$ & $\mathrm{p}=0.008$ \\
\hline PANASneg & $22.42 \pm 9.62$ & $17.68 \pm 10.77$ & $\mathrm{p}=0.007$ \\
\hline $\begin{array}{c}\text { TONI-3 } \\
\text { score }\end{array}$ & $30.84 \pm 9.59$ & $31.95 \pm 8.68$ & $\mathrm{p}=0.347$ \\
\hline
\end{tabular}

Consequently, the results indicated that while undertaking the sequence of physical activities was found to have a positive effect on mood (an area of wellbeing), no significant difference was found on general intelligence. However, it is anticipated that Improvement in 'intelligence' would require significantly longer engagement with an exercise regime than that in an acute study as presently reported. Such longer term effects are something the research team hopes to pursue in the future.

Subjectively, sessions were reported as fun and interesting, particularly given that use of the physical exercise application was not reliant on technological experience. As such, most people rated the programme as enjoyable to very enjoyable in the questionnaire, with the interaction and navigation being easy to very easy to use. In particular, the majority of participants thought the activities were well pitched for someone in the older age group, particularly those who were relatively inactive or involved in little physical activity. Individuals also liked the fact that for people with limited co-ordination or balance, they had the option of either sitting down or standing up to use the programme.

\subsection{Virtual Instructor}

Similarly, comments were very positive about the inclusion of a virtual instructor in the programme, which overall was considered well suited for this age group (e.g. "It was quite good that you think you can do slightly better"). In this regard, criticisms were generally minor, and related to discrepancies in using the pre-recorded videos. This included identifying that the instructor was not always in time with the beat of the music (or the instructions), and did not always accurately replicate the on-screen instructions (e.g. switching the Wii mote into a different hand, see section 4.4). Furthermore, when asked about gender and age preferences, the use of a female instructor was not found to be an issue (although this is based on a very small sample containing very few males). Some participants did stress a preference for a 'fitter' younger looking role model, although these opinions were in a minority. 
More generally, the use of a more representative instructor was found to be reassuring, given that the user was not at peak fitness. Indeed, given that the virtual instructor was apparently of average fitness, it was felt that she could be related to by the user more than a cartoon-type character, such as those demonstrated on game consoles like the Wii Fit.

I'm sure it was deliberate to have, an older person that didn't look like a 20 year old $\mathrm{n}$ a leotard. You know, kinda people you get at gyms, you go there and you think 'yeah well there's no way I can ever emulate those folk' ... so I think that, that was good to have that kind of role model ... I did find the feedback from looking at the instructor useful ... it's a mirror image of course but that's the same as being in a class.

She was may be a 'wee' (little) bit on the heavy side ... but maybe that's to help encourage people ... she looked quite cheerful and happy and I think

In more detail, to highlight some of the main findings from the questionnaire and interview data, a selected summary is given below. These are intended to raise questions rather than present design recommendations.

\subsection{Input Control}

Firstly, while the questionnaires rated the Wii mote as very easy to use, deeper probing in the interviews identified that some found it hard to remember which buttons to press, or when to press them during the exercises. In particular, as part of the routine it was found to be quite taxing to perform an exercise in co-ordination with the button presses. Part of this problem was perceived to relate to the placement of buttons on the control and remembering which button was which, forcing some participants to overly focus on the device rather than concentrate on the on-screen display. As illustrated in table 1, switching between exercises that involved

that was probably more important than her physical attributes,

either pressing $A, B$ or no buttons, meant a fairly high (and ultimately unnecessary) learning curve was required. This was further complicated by the inconsistency between the aerobic section, which relied solely on gesture movement and the muscular exercises which required different button presses. As such, the use of button presses was perceived as being somewhat negative. Clearly, improvements need to be made in the accuracy of the gesture recognition in relation to the co-ordination of activities. Although no one directly indicated the control as cumbersome, it was clear that merely holding an object detracted from exercise performance (including having to shift it from one hand to another between exercises). This suggests the potential benefits of wearable rather than hand-held sensory device(s), particularly with no button pressing requirement.

\subsection{Visual feedback}

Although some participants talked about the advantages of being able to see their own position on-screen while exercising, most

did not initially use it for technique correction. From the questionnaire data, only 5 users indicated that they actively used the video for this purpose. In particular, many users recalled the video to be distracting, either because they did not like to see themselves on-screen, or because they felt the video lagged behind, which meant it was slow in responding to their own physical movements. However, through practice in using the application, it was noticeable that some individuals became more accustomed to looking at the video as a reference point to identify whether their body position or general performance of the activity was correct. As such, it was felt that individual opinions about the live video did not show any consensus. It could be that increased complexity of the exercises performed could make the use of visual feedback more important; however, similarly it may be an unnecessary feature for many of the target users. It will be important to discover more about user perceptions of this feature, as its inclusion uses significant amounts of processing power and imposes technical requirements that could make it unsuitable for the DTV platform.

\begin{abstract}
After the initial horror [laughs]. Seeing yourself in a video, yeah because you can compare your, your body alignment to the models alignment and it gives you a good, understanding of where your body is in relation to the exercise you're doing. So it's very useful from that point of view.
\end{abstract}

\subsection{Clarity of instructions}

Given the previously reported discrepancies between what the instructions described and what was demonstrated by the virtual instructor, and given the novelty of the applications, some individuals struggled to comply. Examples included the concept of briskly marching on the spot between exercises to maintain raised heart rate and avoid 'cooling down'. Although the instructions for this were mainly given between the aerobic activities (including early instructions on the importance of doing this throughout), many simply forgot to do them and stopped in order to pay attention to the next set of instructions. Some users suggested additional prompts, such as an animated walking figure on-screen to remind them when to keep moving.

Similarly, it was also mentioned that at times the instructions were not explicit enough to indicate which hand the user should be holding the Wii Mote in. Although this issue did not have any noticeable 
impact on how the activities were performed, discrepancies in the video demonstration raised the following concerns:

\begin{abstract}
I didn't know whether I was to use one hand or both hands with some of the exercises, it would be useful to say either start with your right hand or ... 'you can do both if you want to', you know because I didn't know some of them whether I was using both hands ...that wasn't explained at the beginning.
\end{abstract}

These examples also further suggest the potential advantages of using a wearable, hands free device, which is likely to have helped simplify the procedure, and make the performance of the exercise the focus of the users' attention, rather than the manipulation of the control device.

\subsection{Quality of the Exercises}

In general, while participants thought that the number and variety of exercises in the programme were appropriate for the study, in order to keep them motivated over a more sustained period of time, there was a consensus that there should be more variation in the available set of exercises. Not only to reduce the monotony of doing the same regimented set on a regular basis, but also to expand on the different sections to accommodate for personal requirements of a more specialised programme.

\begin{abstract}
F or people like me I think it maybe could be a little more demanding ... super fit people just on the bridge of $55 \ldots$ they definitely would want more.
\end{abstract}

\begin{abstract}
All those exercises, and that look, suggest to me over $65 \mathrm{~s}$, definitely that, and I don't see myself in that age group yet, and therefore I, and I think most people think of themselves as fitter than they are.
\end{abstract}

However, in this context it must be recognised that most of the participants were relatively young (average age 62.5 years) and physically active, with many mentioning 'other opportunities' to keep fit rather than having to use a system in their own home. Therefore, had we recruited only physically inactive participants then the outcomes and comments are likely to have been considerably different.

Several participants indicated that mobilisation exercises are particularly important for older adults and that more were required in the programme. There were wrist and shoulder circles included, but suggestions were given to also include hip and neck circling to target the main joints that tend to stiffen with age.

From the interviews it was found that participants thought the 'learnability'of the included exercises was fine for the first time user, or those that were not accustomed to 'exercise class style' activities, although some doubts were raised about the need for the instructions to be quite so detailed.

\subsection{Personalisation}

Feedback during the interviews identified that some participants would have liked more time to perform the exercises, compared to reading the instructions prior to starting the activity. Criticism included too many instructions, or too big a time gap between exercises (despite the marching-onthe-spot activity). This was based on the perception that too many instructions could actually hinder an individual's performance over time. Questions were also raised over the possibility to take a pick and mix approach in terms of being more selective over what exercises were chosen, over a shorter period of exercising time

The background music was perceived to be a useful component of the programme, with comments made that it helped individuals keep in time with the beat, or tempo of the exercise. In this regard, not only was it reported as an important motivational factor, but the light, dance like style of music was described as mood enhancing.

... unless it was a piece of music you hated or had, you know, negative connotations. So I, I think probably it adds to the good effect, yeah I think it's probably a positive thing.

The music I think is very supportive in terms of rhythm, I also think its mood lifting as well and, and keeps you on task.

Related to the criticisms about the problems with the synchronisation and timing of printed and spoken instructions with the movement of the 'virtual instructor', many participants felt it was noticeable when the instructor's pace dropped behind the beat of the music. In this instance, it was unclear whether increasing the intensity of the activity should increase the speed of the instructor's movement on-screen. Furthermore, while the music created real value for the aerobic activities, it was felt to be less significant in the stretching component that required more focused breathing. Mixed reactions were received from participants when asked if they would have liked to customise the music played in the activity programme. On the one hand, a wider variety of music was thought to be important to stop the monotony of hearing the same tune played during prolonged use of the application. Suggestions here included playing the music at different speeds to control the intensity of the exercise. Some participants suggested that this could be based on knowing the beat of the music, or similarly, that 
an option at the side of the user interface could be provided to change the tempo of the current track. Changing the tempo (while retaining the pitch) of the digital music played is technically feasible and could be put under user control as mentioned. However, it could also be adjusted in accordance with the user's heart rate if a device to monitor the user's pulse were integrated into the system. It is possible such a device could be incorporated as part of the wearable 'conrol device' mentioned above.

\section{CONCLUSION \& DISCUSSION}

The unavoidable switch to digital television over the next few years presents a wide spectrum of possibilities. Beyond thecentrality of the medium in people's everyday lives there are potential benefits to integrating a physical fitness programme into a television-based context, such as the possibility of using an electronic programme guide as part of a scheduling system for exercise. However, unlike current commercial offerings, many older adults will require a physical activity plan that is specifically tailored to their needs integrating preventive with therapeutic recommendations for aerobic, muscular strength, endurance, flexibility and balance activities, as well as more specialised therapeutic approaches for particular musculoskeletal problems, or more high impact activities for those people who can tolerate them. Such possibilities seem in line with current UK-based health policies that recognise the importance of increasing life expectancy and general wellbeing [14].

The quantitative results from this study indicate mood significantly improved after the acute trial, while no acute effects were found in cognition. In part, this lack of an acute effect is not a surprise, as a measurable impact on general intelligence would probably require longer term sustained exercise which would lead to improved overall health (and as noted, the current exercises were possibly of too light an intensity to achieve such health benefits). However, it is encouraging to see that small trends in the data indicate possible benefits, with TONI-3 score increasing by about 1 item (see table 3 ). This suggests the potential for measurable cognitive benefits to be obtained from more sustained exercise, which will be incorporated into future research.

Regarding mean heart rate and RPE valves, the majority of exercises were of a light intensity (except for three activities based on heart rate data, and two based on RPE data from the aerobic section, which were classified as moderate intensity) and thus unlikely to produce health benefits. This limitation was reflected in the qualitative feedback where the majority of participants stated that the intensity of exercises was not great enough for them. As such, recommendations for future research include incorporating better mechanisms to guide users in the level of effort required to obtain and maintain health benefits. For example by including body sensors, like a heart rate monitor, in the system it is feasible that physical intensity of the exercises could be visually monitored with feedback to indicate if the user is required to work at a lower or higher intensity to achieve their overall training goals. Similarly, the same information could be utilised within the system to automatically adjust exercise intensity (duration and tempo) in real time and to trigger suitable instructions and prompts. This option may also provide a useful safety mechanism by warning the user should they be dangerously over performing. The inclusion of physiological monitors with wearable motion sensors (possibly on wrists and ankles) and their appropriate integration with the application will play a central role in our future investigation of this system.

The current research has to be put into the context that participants were all reasonably fit and often talked of having 'other opportunities' to keep active. As such, while the study identified that many participants felt that the physical exercise programme was suitable for the target group, on a personal level the duration of activities were not long enough. To reiterate, eachexercise lasted for approximately $30-60$ seconds with the tempo during the warm-up, aerobics and cool-down sections ranging from 130-134 bpm, and between 118-120 bpm for the muscular section. It is likely that had we recruited physically inactive and/or older participants (more representative of the target 'older adults' population) then the outcomes may have been closer to achieving potential health benefits. This will also be considered in the population sampling for future research.

From the interviews it was found that participants thought the amount of instructions in the physical application were fine for first time users. However, some felt that there should be an option to remove some or all of the instructions, which would allow for more time to exercise. Video feedback was also not thought to be an important feature of the application, although it was used for technique correction when individuals became more accustomed to the programme. Alternatively, music was found to be important for time keeping and to enhance mood and motivation. In this regard, useful suggestions from participants included options to customise the music tempo so that they could select to work at a slow, moderate or fast pace. If fully integrated into the system, this could be particularly advantageous in encouraging users to increase the intensity of the exercise when under-performing (or visa-versa).

Further suggestions included expanding the range and style of exercises. In particular, alongside the wrist and shoulder circles it was recommended that 
hip and neck circling should be included to target specific joint movements that tend to stiffen with age. For future implementation, there is also the potential to explore the inclusion of free-weights or resistance bands in the muscle component. This would mean that for any input device used, it should avoid the use of holding a remote or involving button presses where feasible, and rely on more natural forms of body gesture so that the hands are free to hold bands or weights. An example of an input device that does not require the user to hold a remote is a balance board, such as the board used by Nintendo for the NWF, which could be used to detect body movement in the aerobic and muscle sections.

In building on the strengths of using the virtual instructor for older adults, other possibilities include exploring more dynamic means to customise both the design of the character and the real-time feedback required when performing the exercise. Although the gender of the instructor did not appear to be an important issue, some preferences for a younger instructor were expressed. To accommodate for these user variations, and to overcome technical limitations of using a video demonstration, a richer and more integrated (avatar) character is worth pursuing. However, its design must take on board present limitations in the deployment of 3D graphics and native libraries that are not presently specified by open middleware standards, such as by MHP or Java TV [18], as well as the absence of a built-in graphics card in many digital set-top-box receivers. However, the potential of this approach is the greater flexibility it affords for the appearance of the avatar, including the possibility of a range of characters to choose from. It also opens up other possibilities of more flexibility compared to the constraints of prerecorded video, such as user-selected viewing angles and greater adaptability to variations in the exercises and their style being demonstrated.

Finally, to conclude, the main suggestions for improving the physical exercise programme were to a) make the activities more physically intense; b) develop a programme which dynamically progresses with use; c) increase the time length of each activity; d) use feedback that does not require the user to press a button during the exercise; and e) have more personal choice over the instructor and music presented. In line with these research findings our team is currently in collaboration to pursue some of these issues in more detail.

\section{ACKNOWLEDGMENTS}

We would to thank the European Commission for funding this research (Vital Mind FP7-ICT-2007-1) under the 7th framework of 'Ambient Assisted Living' (Challenge 7, Objective 3.7.1.1).

\section{REFERE NCES}

[1] ACSM. 2006. ACSM's guidelines for exercise testing and prescription. Lippincott Williams \& Wilkins.

[2] Allender, S., Foster, C., Scarborough, C., and Rogers, M. 2007. The burden of physical activityrelated ill health in the UK. J. Epidemiol Community $\mathrm{H}$ ealth, 61, 344-348.

[3] Basak C, Boot W. R, Voss M. W, and Kramer A. F. 2008. Can training in a real-time strategy video game attenuate cognitive decline in older adults? J. Psychol Aging, 23(4), 765-777.

[4] Bausch, L. Baran, J., Cahanes, S., and Krug, L. 2008. Physiological responses while playing Nintendo Wii sports. J. Undergraduate Kinesiology Research, 3, 19-25.

[5] BBC. 2009. Computer fitness gets approval. http://news.bbc.co.uk/1/hi/health/8326152.stm

[6] Borg, G. A. V. 1982. Psychophysical bases of perceived exertion, J. Med Sci Sports Exerc, 14(5), 377-381.

[7] Brown, L., Sherbenou, R, J., and Johnsen, S. K. 1997. Test of nonverbal intelligence: a language free measure of cognitive ability (3rd ed.), Austin, PROED.

[8] Goldstein, J., Cajko, L., Oosterbroek, M., Michielsen, M., Van Houten, O., and Salvedera, F. 1997. Videogames and the elderly. J. Social Behavior and Personality, 25, 345 \pm 352 .

[9] Graves, L., Stratton, G., Ridgers, N. D., and Cable, N. T. 2007. Comparison of energy expenditure in adolescents when playing new generation and sedentary computer games: cross sectional study. BMJ Clinical Research, 335, 1282-1284.

[10] MacMillan, F., Kirk, A., Rice M., and Carmichael, A. 2010. Exer-gaming: Using the Nintendo Wii as a physical activity tool in adults aged 55 years and above. Poster presentation at the 3rd International Congress on Physical Activity and Public Health, Toronto.

[11] Nelson, M. E., Rejeski, W. J., Blair, S. N., Duncan, P.W., Judge, J. O., and King, A. C. 2007. Physical activity and public health in older adults: recommendation from the American College of Sports Medicine and the American Heart Association. J. Circulation, 116, 1094-1105.

[12] Resnick, B. 2000. A seven step approach to starting an exercise program for older adults. J. Patient Education and Counseling, 39, 243 \pm 252.

[13] Schuler, P. B., Paradise, S. B., Willoughby, L. A., Kidston, R. W., Sheets, S., Rotunda, R., and CosioLima, L. 2009. The acute effects of Wii-bowling on feeling states and heart rates in older adults. J. Medicine \& Science in Sports \& Exercise, 41(5), 12. [14] Scottish Executive. 2009. The Scottish Health Survey 2008. Edinburgh: Crown Copyright.

[15] Tanaka, H., Monahan, K. D., and Seals, D. R. 2001. Age- predicted maximal heart rate revisited. J. 
Am Coll Cardiol, 37(1), 153-156.

[16] Watson, D., Clark, L. A., and Tellegen, A. 1988. Development and validation of brief measures of positive and negative affect: the PANAS scales. J. Pers Soc Psychol, 54(6), 1063-1070.

[17] Whitcomb, G. R. 1990. Computer games for the elderly. ACM SIGCAS Computers and Society, 20(3).

[18] Ugarte, A., García, I., Ortiz, A., and Oyarzun, D. 2007. User interfaces based on 3D avatars for interactive television. In Proceedings of the 5th European conference of interactive television, Amsterdam, The Netherlands. 107-115. 